\title{
Strongly coupled van der Waals heterostructures for high-performance infrared phototransistor
}

Cite as: Appl. Phys. Lett. 114, 103501 (2019); https://doi.org/10.1063/1.5083685

Submitted: 30 November 2018 . Accepted: 18 January 2019. Published Online: 11 March 2019

Ningning Li, Yao Wen, Ruiqing Cheng, Lei Yin, Feng Wang, Jie Li, Tofik Ahmed Shifa, Liping Feng, Zhenxing Wang, and Jun $\mathrm{He}$

\section{COLLECTIONS}

F This paper was selected as Featured
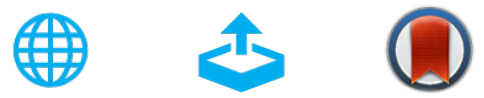

\section{ARTICLES YOU MAY BE INTERESTED IN}

A critical review of recent progress on negative capacitance field-effect transistors Applied Physics Letters 114, 090401 (2019); https://doi.org/10.1063/1.5092684

Graphene and related two-dimensional materials: Structure-property relationships for electronics and optoelectronics

Applied Physics Reviews 4, 021306 (2017); https://doi.org/10.1063/1.4983646

Oriented layered $\mathrm{Bi}_{2} \mathrm{O}_{2}$ Se nanowire arrays for ultrasensitive photodetectors Applied Physics Letters 114, 151104 (2019); https://doi.org/10.1063/1.5094192

Challenge us.

What are your needs for periodic signal detection?

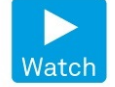

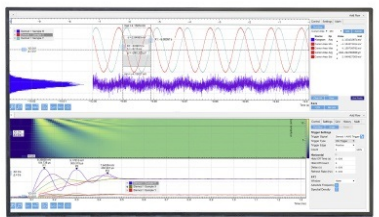

0
- Zurich

- Instruments 


\title{
Strongly coupled van der Waals heterostructures for high-performance infrared phototransistor $\odot$
}

Cite as: Appl. Phys. Lett. 114, 103501 (2019); doi: 10.1063/1.5083685

Submitted: 30 November 2018 - Accepted: 18 January 2019 .

Published Online: 11 March 2019

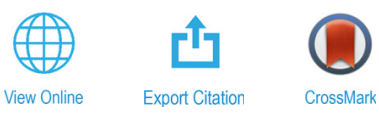

Ningning Li, ${ }^{1,2,3, a)}$ Yao Wen, ${ }^{2,4, a)}$ Ruiqing Cheng, ${ }^{1,2}$ Lei Yin, ${ }^{1,2}$ Feng Wang, ${ }^{1,2}$ Jie Li, $^{1,2}$ Tofik Ahmed Shifa, Liping Feng, ${ }^{5, b)}$ Zhenxing Wang, ${ }^{7, b)}$ and Jun $\mathrm{He}^{7, b)}$

\author{
AFFILIATIONS \\ ${ }^{7}$ CAS Center for Excellence in Nanoscience, CAS Key Laboratory of Nanosystem and Hierarchical Fabrication, National Center for \\ Nanoscience and Technology, Beijing 100190, People's Republic of China \\ ${ }^{2}$ University of Chinese Academy of Sciences, Beijing 100049, People's Republic of China \\ ${ }^{3}$ Sino-Danish Center for Education and Research, Beijing 100190, People's Republic of China \\ ${ }^{4}$ CAS Center for Excellence in Nanoscience, CAS Key Laboratory for Standardization and Measurement for Nanotechnology, \\ National Center for Nanoscience and Technology, Beijing 100190, People's Republic of China \\ ${ }^{5}$ State Key Lab Solidification Processing, College of Materials Science and Engineering, Northwestern Polytechnical University, Xi'an, \\ Shaanxi 710072, People's Republic of China

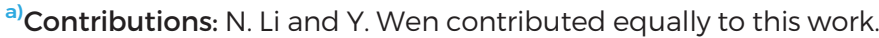 \\ ${ }^{b)}$ Electronic addresses: Ipfeng@nwpu.edu.cn; wangzx@nanoctr.cn; and hej@nanoctr.cn.
}

\begin{abstract}
The interfacial coupling in van der Waals (vdWs) heterostructures can effectively modulate the device performance. In this study, we demonstrate a high performance infrared detector fabricated by direct epitaxial growth of Te nanowires onto a MoS $\mathrm{monolayer}_{2}$ Such directly grown $\mathrm{Te} / \mathrm{MoS}_{2}$ heterostructures show much stronger interfacial coupling than artificially transferred Te/MoS hybrids, as evidenced from their Raman and photoluminescence spectra. The strong vdWs interfacial coupling leads to a high performance infrared detector with both ultrahigh photoresponsivity $\left(>10^{3} \mathrm{~A} / \mathrm{W}\right)$ and rapid response time $\left(\tau_{\text {rising }}=15 \mathrm{~ms}\right)$ at the telecommunication wavelength of $1550 \mathrm{~nm}$. The anti-bipolar and rectification behaviors observed in the strongly coupled grown $\mathrm{Te}-\mathrm{MoS}_{2}$ heterojunction further confirm the effective interfacial coupling. In contrast, the weakly coupled transferred $\mathrm{Te}-\mathrm{MoS}_{2}$ heterojunction that is obtained by artificial transfer exhibits negligible anti-bipolar behavior and slight rectification behavior. These findings indicate that the coupled vdWs hybrid structures have great potential for achieving high performance photodetectors.
\end{abstract}

Published under license by AIP Publishing. https://oi.org/10.1063/1.5083685

Photodiodes based on 2D van der Waals (vdWs) heterostructures, such as $\mathrm{BP} / \mathrm{MoS}_{2}{ }^{1}{ }^{1} \mathrm{BP} / \mathrm{WSe}_{2},{ }^{2}$ and $\mathrm{MoTe}_{2} / \mathrm{MoS}_{2}$, ${ }^{3}$ have been demonstrated in infrared photodetection. Such vdWs heterostructures show a high-quality interface, benefiting from atomically smooth surfaces without dangling bonds. This feature provides a versatile platform to explore their optoelectronic properties. However, they always exhibit very low responsivity because of the absence of internal gain. ${ }^{4-6}$ To significantly improve the gain, hybrid structures based on colloidal quantum dots (CQDs) and 2D materials have been developed. The pioneer work in this framework includes the combination of $\mathrm{PbS}$ QDs with graphene, ${ }^{7,8} \mathrm{MoS}_{2},{ }^{9}$ and $\mathrm{WSe}_{2} .{ }^{10}$ Although high gain was obtained, the poor-quality interfaces, suffering from the weakly interfacial coupling and trap states in the interfaces, lead to very slow response speed. ${ }^{11-13}$ In addition, the loosely contacted interface can broaden the vdWs gap in the interface and further increase the tunnel barrier of carriers from CQDs to 2D materials. ${ }^{14}$ So far, the realization of high responsivity along with rapid response speed has remained an unresolved issue for the above strategies. Fortunately, the strong vdWs coupling can effectively contribute to the interlayer charge transfer and improve the device performance. ${ }^{12}$ Therefore, integrating a highquality and strong-coupled heterointerface into a vdWs hybrid structure is a crucial subject to produce efficient infrared detectors with both fast response speed and high responsivity.

Here, we demonstrate a high performance infrared phototransistor in a coupled hybrid vdWs structure with high-quality interface. Te nanowires, a typical narrow bandgap semiconductor $\left(E_{\mathrm{g}}=0.35 \mathrm{eV}\right),{ }^{15,16}$ were directly grown on the surface of $\mathrm{MoS}_{2}$ nanosheets via vdWs epitaxy. The effective interfacial coupling between the Te layer and 
the $\mathrm{MoS}_{2}$ layer, combined with the strong infrared absorption in Te nanowires, results in ultrahigh photoresponsivity $\left(>10^{3} \mathrm{~A} / \mathrm{W}\right)$ as well as fast response time $\left(\tau_{\text {rising }}=15 \mathrm{~ms}\right)$ at the telecommunication wavelength of $1550 \mathrm{~nm}$. These results indicate that our coupled vdWs hybrid structures have significant potential for the next generation high-performance infrared detectors.

Figure 1(a) presents the schematic illustration of the epitaxial growth of Te nanowires- $\mathrm{MoS}_{2}$ heterostructures. Monolayer $\mathrm{MoS}_{2}$ was first prepared on silicon substrates with $300 \mathrm{~nm}$ thick $\mathrm{SiO}_{2}$ by chemical vapor deposition (CVD). Then, the pre-grown $\mathrm{MoS}_{2}$ was used for the subsequent epitaxial growth of Te nanowires via physical vapor deposition (PVD). (More details are given in the supplementary material.) Te has a typical helical chain-like crystal structure. The atoms in the adjacent chains are combined by weak vdWs forces, ${ }^{17}$ which enable the high-quality vdWs heterointerface between Te and $\mathrm{MoS}_{2}$. The scanning electron microscopy (SEM) image of the grown Te- $\mathrm{MoS}_{2}$ heterostructure is shown in Fig. 1(b). Interestingly, Te nanowires have three specific growth orientations on $\mathrm{MoS}_{2}$ with an angular interval of $60^{\circ}$. Figure $1(\mathrm{c})$ exhibits the histogram statistics of the oriented angle

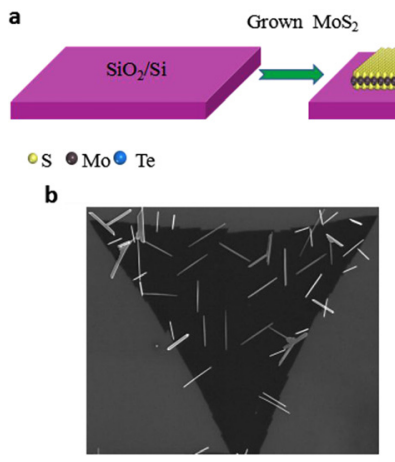

d

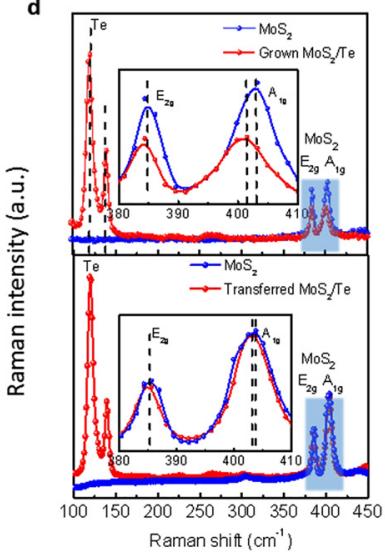

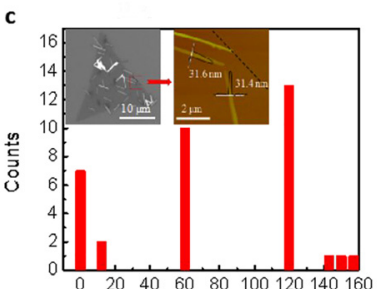

Orientation $\left(^{\circ}\right)$

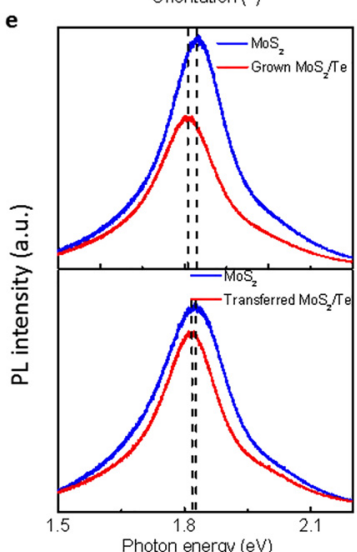

FIG. 1. (a) Schematic illustration of two-step epitaxial growth of Te nanowires-MoS heterostructures. (b) SEM image of epitaxial Te nanowires-MoS $\mathrm{S}_{2}$ heterostructures. The scale bar is $5 \mu \mathrm{m}$. (c) The histogram statistics of the orientation distribution of Te nanowires obtained from the left inset. Left inset: the optical microscope (OM) image of Te nanowires-MoS 2 heterostructure. Right inset: the corresponding AFM image and height profiles. (d) Raman and (e) PL spectra of individual $\mathrm{MoS}_{2}$, grown Te nanowires-MoS $\mathrm{S}_{2}$ and transferred Te nanowires-MoS 2 heterostructures. The inset in figure (d) shows the details of the marked blue region. distribution of Te nanowires, in which $86 \%$ of the nanowires follow three specific directions. Compared to the previous hybrid QDs system with a random distribution, the oriented Te nanowires have much stronger interaction with $\mathrm{MoS}_{2} .{ }^{18}$ The chemical states and the composition of nanowires were confirmed by X-ray photoelectron spectroscopy (XPS) analysis. As shown in Fig. S1 in the supplementary material, the peaks at 584.1 and $573.7 \mathrm{eV}$ correspond to $\mathrm{Te} 3 \mathrm{~d}_{3 / 2}$ and $3 \mathrm{~d}_{5 / 2}$, while the other two peaks are attributed to tellurium oxide produced by surface oxidation. ${ }^{19,20}$ The thickness of Te nanowires and $\mathrm{MoS}_{2}$ nanosheets was characterized by atomic force microscopy (AFM). The right inset of Fig. 1(c) shows the AFM image and the height profile of two typical Te nanowires, in which the thickness is identified to be 31.4 and $31.6 \mathrm{~nm}$. The AFM image of monolayer $\mathrm{MoS}_{2}$ prepared by CVD is shown in Fig. S2 in the supplementary material. To characterize the interfacial coupling between the Te layer and the $\mathrm{MoS}_{2}$ layer, Raman and photoluminescence (PL) spectroscopy were conducted. Two types of Te-MoS 2 interfaces were constructed: (i) a Te- $\mathrm{MoS}_{2}$ heterostructure prepared by direct epitaxial growth and (ii) a Te- $\mathrm{MoS}_{2}$ heterostructure fabricated by artificially stacking Te nanowires onto $\mathrm{MoS}_{2}$. Raman spectroscopy is a sensitive tool to accurately characterize the interlayer coupling in vdWs heterostructures. ${ }^{21}$ Figure 1(d) shows the Raman spectrum of individual $\mathrm{MoS}_{2}$ and the two types of $\mathrm{Te}-\mathrm{MoS}_{2}$ vdWs heterostructures. In the as-grown $\mathrm{Te}-\mathrm{MoS}_{2}$ vdWs heterostructures, the $\mathrm{A}_{1}\left(\sim 119.6 \mathrm{~cm}^{-1}\right)$ and $\mathrm{E}_{1}\left(\sim 139.1 \mathrm{~cm}^{-1}\right)$ modes of $\mathrm{Te}^{22,23}$ as well as the out-of-plane $\mathrm{A}_{\mathrm{lg}}\left(\sim 400.9 \mathrm{~cm}^{-1}\right)$ and in-plane $\mathrm{E}_{2 \mathrm{~g}}\left(\sim 384.2 \mathrm{~cm}^{-1}\right)$ modes of monolayer $\mathrm{MoS}_{2}$ are observed. Compared with the individual monolayer $\operatorname{MoS}_{2}\left(\mathrm{~A}_{1 \mathrm{~g}} \sim 403.4 \mathrm{~cm}^{-1}\right.$ and $E_{2 g} \sim 384.9 \mathrm{~cm}^{-1}$ ), the $A_{1 g}$ and $E_{2 g}$ peaks in overlapped $\mathrm{MoS}_{2}$ were red-shifted, indicating the electron transfer from the Te layer to the $\mathrm{MoS}_{2}$ layer at the interface. ${ }^{4,24,25} \mathrm{~A}_{1 \mathrm{~g}}$ shows a distinct red shift $\left(\sim 2.5 \mathrm{~cm}^{-1}\right)$, but only a slight red shift is observed in the $\mathrm{E}_{2 \mathrm{~g}}$ peak; this is because electrons are coupled more tightly with $\mathrm{A}_{1 \mathrm{~g}}$ photons than $\mathrm{E}_{2 \mathrm{~g}}$ photons. ${ }^{25}$ And, the decrease in $\mathrm{A}_{1 \mathrm{~g}}$ peak intensity can be attributed to the stacking of Te nanowires on $\mathrm{MoS}_{2}$, which can restrain its out-of-plane vibration. ${ }^{24}$ But, for artificially transferred Te-MoS 2 heterostructures, some polymer residues were left and ambient gases were trapped at the interface during the transfer process, forming a weak interface, and thus the changes in the Raman frequencies are negligible. The corresponding PL spectrum is depicted in Fig. 1(e). The exciton peak A is prominently redshifted and PL intensity is reduced in the grown heterostructure compared with individual $\mathrm{MoS}_{2}$, which indicate the efficient charge transfer at the interface of $\mathrm{Te}$ and $\mathrm{MoS}_{2}{ }^{25,26}$ The excess electrons transferred from Te can effectively combine with excitons in $\mathrm{MoS}_{2}$ and form the negative trions, thus lowering the charge recombination and peak intensity of exciton A. But only a slight change is observed in the transferred Te- $\mathrm{MoS}_{2}$ heterostructure due to weak interfacial coupling.

Figure 2(a) shows the schematic diagram of the hybrid phototransistor, in which Te nanowires are designed as the infrared absorber layer and $\mathrm{MoS}_{2}$ is used as the conducting channel. The electrodes were fabricated by standard electron-beam lithography (EBL) and thermal evaporation of $\mathrm{Cr} / \mathrm{Au}(10 / 60 \mathrm{~nm})$. The gate voltage was applied to the heavily n-doped silicon substrate. The length and the width of the channel in our device are $3.8 \mu \mathrm{m}$ and $21.2 \mu \mathrm{m}$, respectively. The corresponding power density-dependent transfer curves $\left(I_{\mathrm{ds}}-V_{\mathrm{gs}}\right)$ at the $1550 \mathrm{~nm}$ laser are given in Fig. 2(b). Under laser illumination, there is a noticeable increase in the drain current even with an ultralow power 
a
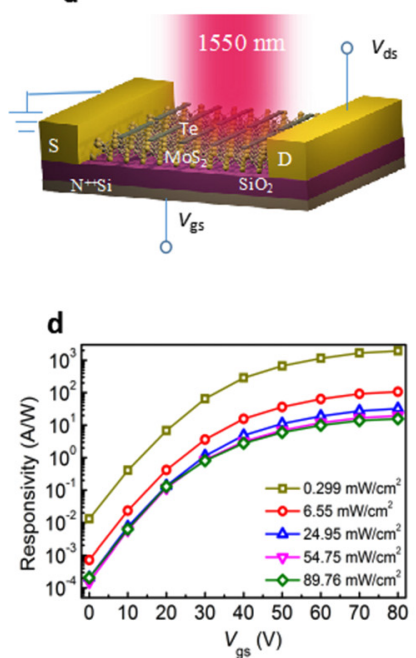

b $_{5.0}$

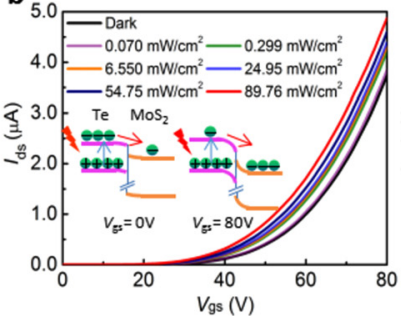

e

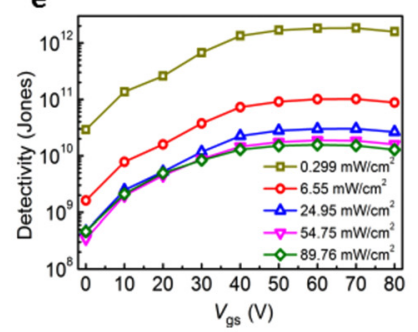

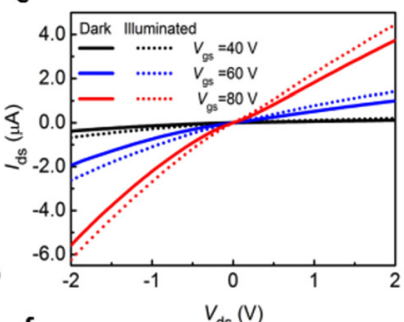

f

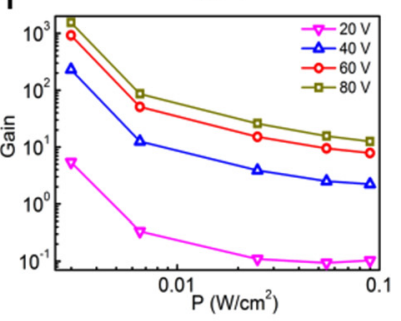

FIG. 2. (a) Schematic of our Te nanowires- $\mathrm{MoS}_{2}$ hybrid phototransistor. Te nanowires and the $\mathrm{MoS}_{2}$ nanosheet act as the infrared absorber layer and the transport channel, respectively. (b) Transfer characteristic $\left(I_{\mathrm{ds}}-V_{\mathrm{gs}}\right)$ curves under illumination of a $1550 \mathrm{~nm}$ laser with different power densities $\left(V_{d s}=2 \mathrm{~V}\right)$, the inset shows the energy band diagrams at the junction formed by Te and $\mathrm{MoS}_{2}$ under $V_{\mathrm{gs}}=0 \mathrm{~V}$ and $80 \mathrm{~V}$. (c) Output characteristic $\left(I_{\mathrm{ds}}-V_{\mathrm{ds}}\right)$ curves under dark and illumination states. (d) Responsivity and (e) detectivity of the heterostructures are plotted as a function of $V_{g s}$. (f) Plots of photoconductive gain $(\mathrm{G})$ versus power density at different $V_{\mathrm{gs}}$. density of $0.07 \mathrm{~mW} / \mathrm{cm}^{2}$ at $80 \mathrm{~K}$. CVD-grown $\mathrm{MoS}_{2}$ indeed has much various structural defects due to growth process imperfections. However, the photoresponse of the individual $\mathrm{MoS}_{2}$ device under a $1550 \mathrm{~nm}$ laser was also measured in Fig. S3(a) in the supplementary material, which is negligible compared with the $\mathrm{Te} / \mathrm{MoS}_{2}$ hybrid device due to the intrinsic band gap limitation, and no obvious damage to $\mathrm{MoS}_{2}$ is observed after growth of Te nanowires [Figs. S3(b) and $\mathrm{S} 3$ (c) in the supplementary material]. Moreover, an individual $\mathrm{Te}$ nanowire shows obvious photoresponse at $1550 \mathrm{~nm}$ (Fig. S4 in the supplementary material). Thus, the photo-generated carriers mainly originate from Te nanowires in our hybrid device. Driven by the vertical built-in field between Te and $\mathrm{MoS}_{2}$, photo-generated electrons in Te nanowires are transferred to the $\mathrm{MoS}_{2}$ channel, while the holes are confined in the Te nanowires due to the potential barrier at the interface. ${ }^{27}$ This directly leads to the increase in the $\mathrm{MoS}_{2}$ channel current. The obvious photoresponse is also reflected in the output curves, as displayed in Fig. 2(c). The relationship of photocurrent $\left(I_{\mathrm{ph}}=I_{\text {light }}\right.$ $\left.-I_{\text {dark }}\right)$ and power densities at different gate voltages is plotted in Fig. S5 in the supplementary material, in which $\mathrm{I}_{\mathrm{ph}}$ increases monotonically with the power densities.

To accurately evaluate the device performance, responsivity $(R)$, which is defined as

$$
R=\frac{I_{p h}}{P S},
$$

where $P$ is the power density and $S$ is the effective illumination area, was extracted to further quantify the photoresponse performance. As displayed in Fig. 2(d), responsivity increases with the gate voltage, and the maximum responsivity of $10^{3} \mathrm{~A} / \mathrm{W}$ is obtained under $V_{\mathrm{gs}}=80 \mathrm{~V}$ and $P=0.299 \mathrm{~mW} / \mathrm{cm}^{2}$. Owing to the electric field screening effect from the underneath $\mathrm{MoS}_{2}$, the applied $V_{\mathrm{gs}}$ mainly regulates the Fermi level of $\mathrm{MoS}_{2}$ and leaves the upper Te unaffected. ${ }^{28,29}$ Since $\mathrm{MoS}_{2}$ is already in the depletion region at $V_{\mathrm{gs}}=0 \mathrm{~V}$, the Fermi level is located at the same position at negative and zero gate voltages. As shown in the inset of Fig. 2(b), at lower $\mathrm{V}_{\mathrm{gs}}$, the relatively weak built-in electric field formed between $\mathrm{Te}$ and $\mathrm{MoS}_{2}$ enables photogenerated electron transfer from Te to $\mathrm{MoS}_{2}$ and generate smaller photocurrent. When $V_{\mathrm{gs}}$ increases, the Fermi level of $\mathrm{MoS}_{2}$ is lifted up, and the enhanced vertical built-in field effectively facilitates the transfer of electrons from Te to $\mathrm{MoS}_{2}$, resulting in the enhancement of photocurrent and responsivity. As $V_{\mathrm{gs}}$ increases to $80 \mathrm{~V}, R$ gradually approaches a saturated state, which can be attributed to the fast recombination between photogenerated holes and excess electrons in $\mathrm{MoS}_{2}$, and the saturation of carrier mobility in $\mathrm{MoS}_{2} \cdot{ }^{30}$ The relationship between responsivity and power density is presented in Fig. S6 in the supplementary material. As can be seen, the responsivity decreases as the power density increases. Under higher illumination intensity, the increase in the number of separated photo-generated carriers produces an electric field against the direction of the built-in electric field. The diminished built-in electric field then accelerates the carriers' recombination, which in turn leads to the decrement of responsivity. The specific detectivity $\left(D^{*}\right)$ is another essential parameter for photodetectors. It can be calculated as

$$
D^{*}=R \sqrt{\frac{S}{2 q I_{\text {dark }}}}=\frac{I_{p h}}{\sqrt{I_{\text {dark }}}} \cdot \frac{1}{P \sqrt{2 q S}},
$$

in which $R, S, q$, and $I_{\mathrm{dark}}$ are the responsivity, the effective illumination area, the electron charge, and the dark current, respectively. As shown in Fig. 2(e), the maximal detectivity of $10^{12}$ Jones is obtained at $V_{\mathrm{gs}}=80 \mathrm{~V}$ and $P=0.299 \mathrm{~mW} / \mathrm{cm}^{2}$. In addition, our hybrid device exhibits a calculated high photoconductive gain $(\mathrm{G})^{31}$ of over $10^{3}$, which is defined as

$$
G=\frac{R h c}{q \lambda},
$$

where $h$ is the Planck constant, $\mathrm{c}$ is the light velocity, and $\lambda$ is the light wavelength, as shown in Fig. 2(f), which is suggestive of the fact that more than $10^{3}$ electrons can be achieved per photon at $1550 \mathrm{~nm}$. Figure 3(a) presents the dynamic photocurrent response for a Te- $\mathrm{MoS}_{2}$ hybrid phototransistor at a $1550 \mathrm{~nm}$ laser pulse with power densities of 6.55 and $24.95 \mathrm{~mW} / \mathrm{cm}^{2}$. An amplified photoresponse 

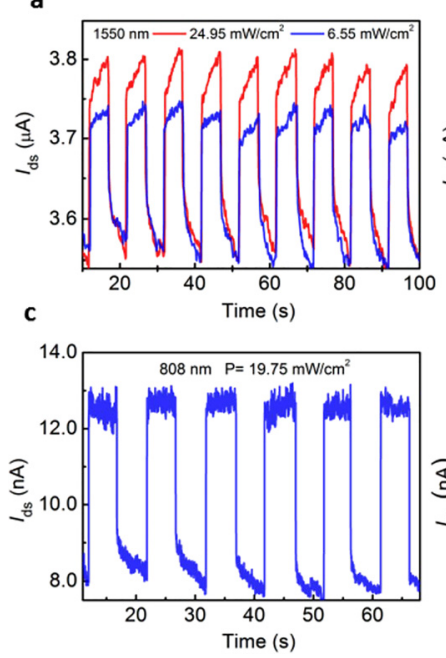

b
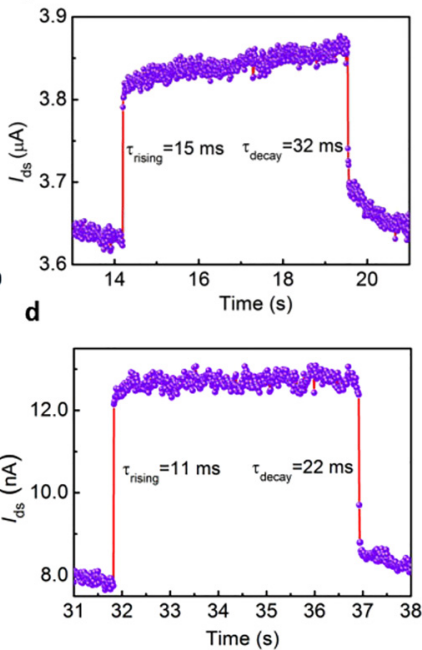

FIG. 3. (a) Dynamic photoresponse of Te nanowires-MoS 2 heterostructures was measured at $1550 \mathrm{~nm}$ with different power densities of 24.95 and $6.55 \mathrm{~mW} / \mathrm{cm}^{2}$ $\left(V_{\mathrm{ds}}=2 \mathrm{~V}, \quad V_{\mathrm{gs}}=80 \mathrm{~V}\right)$. (b) Temporal response shows $\tau_{\text {rising }}=15 \mathrm{~ms}$ and $\tau_{\text {decay }}=32 \mathrm{~ms}$ at $1550 \mathrm{~nm}$. (c) Dynamic photoresponse at $808 \mathrm{~nm}$ with power density $P=19.75 \mathrm{~mW} / \mathrm{cm}^{2}\left(V_{\mathrm{ds}}=2 \mathrm{~V}, V_{\mathrm{gs}}=20 \mathrm{~V}\right)$. (d) Temporal response shows $\tau_{\text {rising }}=11 \mathrm{~ms}$ and $\tau_{\text {decay }}=22 \mathrm{~ms}$ at $808 \mathrm{~nm}$.

cycle is given in Fig. 3(b); it exhibits a fast photoresponse speed ( $\tau_{\text {rising }}=15 \mathrm{~ms}$ and $\tau_{\text {decay }}=32 \mathrm{~ms}$ ), which is superior to the previously reported CQDs-based hybrid photodetectors. ${ }^{7-10}$ Similarly, photoresponse of the Te- $\mathrm{MoS}_{2}$ hybrid device to $808 \mathrm{~nm}$ was also measured. A stable photo-switching behavior can also be monitored at an incident power density of $19.75 \mathrm{~mW} / \mathrm{cm}^{2}$, as shown in Fig. 3(c). Figure 3(d) exhibits a faster response speed $\left(\tau_{\text {rising }}=11 \mathrm{~ms}\right.$ and $\left.\tau_{\text {decay }}=22 \mathrm{~ms}\right)$. As shown in Fig. S7(a) in the supplementary material, the rising and decay times can reach $4.59 \mathrm{~ms}$ and $9.16 \mathrm{~ms}$, respectively, with the assistance of an oscilloscope. In addition, we also studied the effect of surface coverage of $\mathrm{Te}$ nanowires on photoresponse performance. The density of Te nanowires exhibited the tendency to increase with the reaction time (Fig. S8 in the supplementary material). As shown in Fig. S9(a) in the supplementary material, when the surface coverage is $30 \%$, the rising and decay times of the $\mathrm{Te} / \mathrm{MoS}_{2}$ hybrid device are 23 and $39 \mathrm{~ms}$, respectively. Faster response times $\left(\tau_{\text {rising }}=23 \mathrm{~ms}\right.$, $\tau_{\text {decay }}=32 \mathrm{~ms}$ and $\tau_{\text {rising }}=15 \mathrm{~ms}, \tau_{\text {decay }}=32 \mathrm{~ms}$ ) are obtained for the device with surface coverages of $50 \%$ and $60 \%$, as observed in Figs. S9(b) and S9(c) in the supplementary material. The results indicate that the surface coverage dependence of response time is negligible. The responsivity of the device with different surface coverages was also calculated. As shown in Fig. S9(d) in the supplementary material, the responsivity shows obvious enhancement with the increase of the density of Te nanowires.

The comparison of individual $\mathrm{Te}$ and $\mathrm{Te} / \mathrm{MoS}_{2}$ heterostructure devices is given in Fig. S10 in the supplementary material. As shown in Figs. S10(a) and S10(b), the individual Te and Te/ $\mathrm{MoS}_{2}$ devices possess similar magnitudes of photocurrent and $\mathrm{I}_{\mathrm{ph}}$ increases monotonically with power density P. For response time, as shown in Figs. S10(c) and $\mathrm{S} 10(\mathrm{~d})$, compared with $\mathrm{Te} / \mathrm{MoS}_{2}$ devices $\left(\tau_{\text {rising }}=15 \mathrm{~ms}\right.$ and $\tau_{\text {decay }}=32 \mathrm{~ms}$ ), individual Te shows a much slower response speed

( $\tau_{\text {rising }}=110 \mathrm{~ms}, \tau_{\text {decay }}=170 \mathrm{~ms}$ ). This can be attributed to the fast recombination between photogenerated electrons and holes in Te. However, for $\mathrm{Te} / \mathrm{MoS}_{2}$ devices, the introduction of a vertical built-in field facilitates the rapid separation between photogenerated electrons and holes in Te. Besides, the strong vdWs coupling between the Te layer and the $\mathrm{MoS}_{2}$ layer can effectively contribute to the interlayer charge transfer from Te to $\mathrm{MoS}_{2}$, resulting in rapid response speed. ${ }^{13}$ Due to the intrinsic small light absorption area of the Te nanowire, the responsivity of the individual Te device is slightly larger than the $\mathrm{Te} /$ $\mathrm{MoS}_{2}$ hybrid device [Figs. S10(e) and S10(f)]. However, it is worth noting that the individual Te photodetector suffers from very high dark current $\left[10^{-5}-10^{-6} \mathrm{~A}\right.$, Fig. S10(h) $]$, leading to low detectivity $\left[\sim 10^{10}\right.$ Jones, Fig. S10(f)] and a light switching ratio [ 1.5, Fig. S10(h)]. In contrast, the $\mathrm{Te} / \mathrm{MoS}_{2}$ device shows higher detectivity $\left[\sim 10^{12}\right.$ Jones, Fig. $S 10(\mathrm{e})]$ and a light switching ratio $[\sim 100$, Fig. S10(g)] benefiting from $\mathrm{MoS}_{2}$ as a conducting channel. Overall, the performance of the $\mathrm{Te} / \mathrm{MoS}_{2}$ device is superior to the individual Te device for infrared photodetection. Moreover, a comparison with previously reported infrared photodetectors is presented in Table I in the supplementary material. The overall performance of our device is found to be outstanding among the 2D infrared photodetectors. Compared with previous work, our work has made the following improvements: firstly, for the previous QDs-based hybrid photodetector, the traps at the interface can prolong the lifetime of the photocarriers and further severely slow the response speed. And, the interlayer charge transfer can be partially blocked due to the weak interfacial coupling. ${ }^{32,33}$ Benefiting from the high-quality vdWs interface and effective interfacial coupling in our device, the response speed has been greatly improved. Then, for previous photodiodes, the potential barrier generated at the junction prevents the carrier recirculation and further restricts the gain, ${ }^{6}$ in contrast to these photodiodes, $\mathrm{MoS}_{2}$ operates in the conductor mode instead of the diode mode in our device, which promotes the responsivity. ${ }^{34}$

To further explore the origin of the outstanding photodetection ability in our hybrid device, the interfacial interaction modulated by the vdWs coupling was studied in the grown and the transferred $\mathrm{Te}-\mathrm{MoS}_{2}$ heterojunction. The means of deposition of the Te layer is crucial to the operation of the device. In the grown Te- $\mathrm{MoS}_{2}$ heterojunction diode, significant anti-bipolar behavior ${ }^{35-38}$ is observed when applying a reverse bias, as displayed in Fig. 4(a). As a result, with the moderate carrier density of both Te and $\mathrm{MoS}_{2}$, the maximum value of drain current is obtained when $V_{\mathrm{gs}} \approx 20 \mathrm{~V}$. And, the grown heterojunction also shows obvious rectification characteristic, and the highest rectification ratio of $10^{3}$ is obtained under $V_{\mathrm{gs}}=80 \mathrm{~V}$, as shown in Fig. 4(b). But for the transferred Te- $\mathrm{MoS}_{2}$ heterojunction, no obvious antibipolar behavior is observed [Fig. 4(c)], and these two semiconductors fail to produce an efficient rectifying heterojunction [Fig. 4(d)], ${ }^{14}$ which also indicates that the rectification phenomenon comes mainly from the heterojunction region rather than the contact region. Based on the above discussion, the effective interfacial coupling in $\mathrm{Te}-\mathrm{MoS}_{2}$ heterostructures directly grown by CVD contributes to the rapid charge transfer at the interface, accompanied by the high infrared absorption in Te, leading to the outstanding photodetection ability of our device.

In conclusion, we demonstrated an infrared phototransistor based on a coupled Te- $\mathrm{MoS}_{2}$ hybrid structure, in which Te nanowires are directly grown on $\mathrm{MoS}_{2}$ via vdWs epitaxy. Benefiting from the 

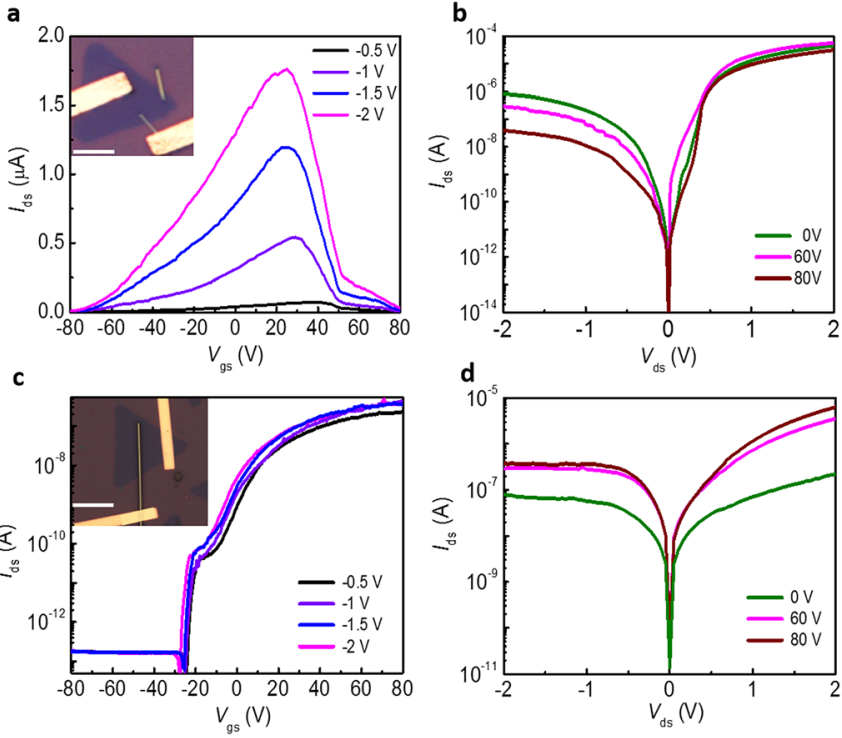

FIG. 4. (a) Transfer characteristic $\left(I_{\mathrm{ds}}-V_{\mathrm{gs}}\right)$ measurements of the grown Te-MoS heterojunction diode. The inset shows the OM image of the grown $\mathrm{Te}_{-} \mathrm{MoS}_{2}$ heterojunction, the scale bar is $5 \mu \mathrm{m}$. (b) Output characteristic $\left(I_{\mathrm{ds}}-V_{\mathrm{ds}}\right)$ measurements of the grown $\mathrm{Te}-\mathrm{MoS}_{2}$ heterojunction diode under different back-gated voltages $V_{\mathrm{gs}}$. (c) Transfer characteristics $\left(I_{\mathrm{ds}}-V_{\mathrm{gs}}\right)$ of the artificially transferred Te-MoS $\mathrm{S}_{2}$ heterojunction diode. The inset shows the OM image of the artificially transferred Te-MoS 2 heterojunction, the scale bar is $5 \mu \mathrm{m}$. (d) Output characteristic $\left(I_{\mathrm{ds}}-V_{\mathrm{ds}}\right)$ measurements of the transferred $\mathrm{Te}-\mathrm{MoS}_{2}$ heterojunction diode.

strong vdWs coupling between the Te layer and the $\mathrm{MoS}_{2}$ layer, the device exhibits not only ultrahigh photoresponsivity $\left(>10^{3} \mathrm{~A} / \mathrm{W}\right)$ but also a rapid response time $\left(\tau_{\text {rising }}=15 \mathrm{~ms}\right)$ at the telecommunication wavelength of $1550 \mathrm{~nm}$. Raman and PL studies indicate the effective interfacial coupling in the grown $\mathrm{Te}-\mathrm{MoS}_{2}$ heterojunction. And, the anti-bipolar and rectification behaviors observed in the strongly coupled grown Te- $\mathrm{MoS}_{2}$ heterojunction further confirmed the existence of interfacial coupling. By comparison, the weakly coupled transferred $\mathrm{Te}-\mathrm{MoS}_{2}$ heterojunction exhibits negligible anti-bipolar behavior and slight rectification behavior. These results indicate that the coupled vdWs hybrid structures have significant potential for the next generation high-performance infrared detectors.

See supplementary material for more experimental details and device characterization.

This work was supported by the Ministry of Science and Technology of China (No. 2016YFA0200700), National Natural Science Foundation of China (Nos. 61625401, 61474033, 61574050, and 11674072), Strategic Priority Research Program of the Chinese Academy of Sciences (Grant No. XDA09040201), and CAS Key Laboratory of Nanosystem and Hierarchical Fabrication. The authors also gratefully acknowledge the support of Youth Innovation Promotion Association CAS.

\section{REFERENCES}

${ }^{1}$ L. Ye, H. Li, Z. Chen, and J. Xu, ACS Photonics 3, 692 (2016).

${ }^{2}$ L. Ye, P. Wang, W. Luo, F. Gong, L. Liao, T. Liu, L. Tong, J. Zang, J. Xu, and W. Hu, Nano Energy 37, 53 (2017).
${ }^{3}$ K. Zhang, T. Zhang, G. Cheng, T. Li, S. Wang, W. Wei, X. Zhou, W. Yu, Y. Sun, P. Wang, D. Zhang, C. Zeng, X. Wang, W. Hu, H. J. Fan, G. Shen, X. Chen, X. Duan, K. Chang, and N. Dai, ACS Nano 10, 3852 (2016).

${ }^{4}$ X. Zhou, X. Hu, S. Zhou, H. Song, Q. Zhang, L. Pi, L. Li, H. Li, J. Lu, and T. Zhai, Adv. Mater. 30, 1703286 (2018).

${ }^{5}$ M. Buscema, J. O. Island, D. J. Groenendijk, S. I. Blanter, G. A. Steele, H. S. van der Zant, and A. Castellanos-Gomez, Chem. Soc. Rev. 44, 3691 (2015).

${ }^{6}$ D. Kufer and G. Konstantatos, ACS Photonics 3, 2197 (2016).

${ }^{7}$ D. Zhang, L. Gan, Y. Cao, Q. Wang, L. Qi, and X. Guo, Adv. Mater. 24, 2715 (2012).

${ }^{8}$ Z. Sun, Z. Liu, J. Li, G. A. Tai, S. P. Lau, and F. Yan, Adv. Mater. 24, 5878 (2012).

${ }^{9}$ D. Kufer, I. Nikitskiy, T. Lasanta, G. Navickaite, F. H. Koppens, and G. Konstantatos, Adv. Mater. 27, 176 (2015).

${ }^{10} \mathrm{C}$. Hu, D. Dong, X. Yang, K. Qiao, D. Yang, H. Deng, S. Yuan, J. Khan, Y. Lan, H. Song, and J. Tang, Adv. Funct. Mater. 27, 1603605 (2017).

${ }^{11}$ W. Xu, W. Liu, J. F. Schmidt, W. Zhao, X. Lu, T. Raab, C. Diederichs, W. Gao, D. V. Seletskiy, and Q. Xiong, Nature 541, 62 (2017).

${ }^{12}$ C. Jin, E. Y. Ma, O. Karni, E. C. Regan, F. Wang, and T. F. Heinz, Nat. Nanotechnol. 13, 994 (2018).

${ }^{13}$ X. Hong, J. Kim, S.-F. Shi, Y. Zhang, C. Jin, Y. Sun, S. Tongay, J. Wu, Y. Zhang, and F. Wang, Nat. Nanotechnol. 9, 682 (2014).

${ }^{14} \mathrm{~V}$. Adinolfi and E. H. Sargent, Nature 542, 324 (2017).

${ }^{15}$ Y. Du, G. Qiu, Y. Wang, M. Si, X. Xu, W. Wu, and P. D. Ye, Nano Lett. 17, 3965 (2017).

${ }^{16}$ X. Huang, J. Guan, Z. Lin, B. Liu, S. Xing, W. Wang, and J. Guo, Nano Lett. 17, 4619 (2017).

${ }^{17}$ Z. He, Y. Yang, J. W. Liu, and S. H. Yu, Chem. Soc. Rev. 46, 2732 (2017).

${ }^{18}$ A. R. Jang, S. Hong, C. Hyun, S. I. Yoon, G. Kim, H. Y. Jeong, T. J. Shin, S. O. Park, K. Wong, S. K. Kwak, N. Park, K. Yu, E. Choi, A. Mishchenko, F. Withers, K. S. Novoselov, H. Lim, and H. S. Shin, Nano Lett. 16, 3360 (2016).

${ }^{19}$ H. S. White, A. J. Rlcco, and M. S. Wrighton, J. Phys. Chem. 87, 5140 (1983).

${ }^{20}$ M. K. Bahl, R. L. Watson, and K. J. Irgolic, J. Chem. Phys. 66, 5526 (1977).

${ }^{21}$ K. Zhang, S. Hu, Y. Zhang, T. Zhang, X. Zhou, Y. Sun, T.-X. Li, H. J. Fan, G. Shen, X. Chen, and N. Dai, ACS Nano 9, 2704 (2015).

${ }^{22}$ Q. S. Wang, M. Safdar, K. Xu, M. Mirza, Z. X. Wang, and J. He, ACS Nano 8, 7497 (2014).

${ }^{23}$ A. S. Pine and G. Dresselhaus, Phys. Rev. B 4, 356 (1971).

${ }^{24}$ D. H. Luong, H. S. Lee, G. P. Neupane, S. Roy, G. Ghimire, J. H. Lee, Q. A. Vu, and Y. H. Lee, Adv. Mater. 29, 1701512 (2017).

${ }^{25}$ J. D. Lin, C. Han, F. Wang, R. Wang, D. Xiang, S. Qin, X.-A. Zhang, L. Wang, H. Zhang, A. T. S. Wee, and W. Chen, ACS Nano 8, 5323 (2014).

${ }^{26}$ S. Mouri, Y. Miyauchi, and K. Matsuda, Nano Lett. 13, 5944 (2013).

${ }^{27}$ Y. Liu, F. Wang, X. Wang, X. Wang, E. Flahaut, X. Liu, Y. Li, X. Wang, Y. Xu, Y. Shi, and R. Zhang, Nat. Commun. 6, 8589 (2015).

${ }^{28}$ A. Castellanos-Gomez, E. Cappelluti, R. Roldan, N. Agrait, F. Guinea, and G. Rubio-Bollinger, Adv. Mater. 25, 899 (2013).

${ }^{29}$ N. Huo and G. Konstantatos, Nat. Commun. 8, 572 (2017).

${ }^{30}$ G. Fiori, B. N. Szafranek, G. Iannaccone, and D. Neumaier, Appl. Phys. Lett. 103, 233509 (2013).

${ }^{31}$ F. H. Koppens, T. Mueller, P. Avouris, A. C. Ferrari, M. S. Vitiello, and M. Polini, Nat. Nanotechnol. 9, 780 (2014).

${ }^{32}$ B. Y. Zhang, T. Liu, B. Meng, X. Li, G. Liang, X. Hu, and Q. J. Wang, Nat. Commun. 4, 1811 (2013).

${ }^{33}$ J. Miao, W. Hu, N. Guo, Z. Lu, X. Liu, L. Liao, P. Chen, T. Jiang, S. Wu, J. C. Ho, L. Wang, X. Chen, and W. Lu, Small 11, 936 (2015).

${ }^{34}$ Z. Chen, X. Li, J. Wang, L. Tao, M. Long, S. J. Liang, L. K. Ang, C. Shu, H. K. Tsang, and J. B. Xu, ACS Nano 11, 430 (2017).

${ }^{35}$ M. H. Doan, Y. Jin, S. Adhikari, S. Lee, J. Zhao, S. C. Lim, and Y. H. Lee, ACS Nano 11, 3832 (2017).

${ }^{36}$ A. Nourbakhsh, A. Zubair, M. S. Dresselhaus, and T. Palacios, Nano Lett. 16, 1359 (2016).

${ }^{37}$ F. Wang, L. Yin, Z. X. Wang, K. Xu, F. M. Wang, T. A. Shifa, Y. Huang, C. Jiang, and J. He, Adv. Funct. Mater. 26, 5499 (2016).

${ }^{38}$ Y. T. Lee, P. J. Jeon, J. H. Han, J. Ahn, H. S. Lee, J. Y. Lim, W. K. Choi, J. D. Song, M.-C. Park, S. Im, and D. K. Hwang, Adv. Funct. Mater. 27, 1703822 (2017). 